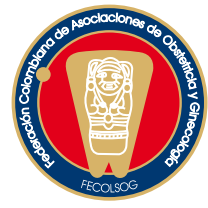

INVESTIGACIÓN ORIGINAL

\title{
ASOCIACIÓN ENTRE EL ÍNDICE DE MASA CORPORAL Y LOS RESULTADOS PERIOPERATORIOS Y POSOPERATORIOS EN PACIENTES SOMETIDAS A HISTERECTOMÍA LAPAROSCÓPICA TOTAL. MEDELLÍN (COLOMBIA) 2002-2008
}

\section{The association between body mass index and perioperative and postoperative outcomes in patients undergoing total laparoscopic lhysterectomy. Medellin, Colombia 2002-2008}

Juan D. Castañeda, M.D. *, José F. De Los Ríos, M.D. *, Gustavo A. Calle, M.D. *, Eduardo Serna, M.D. *, Ricardo A. Vásquez, M.D.*, Ana M. Mejía**, Adriana M. Arango, M.D.***, Carlos A. Buitrago, M.D.****

Recibido: junio 18/09 - Aceptado: mayo 23/10

\section{RESUMEN}

Objetivos: evaluar la posible asociación entre el mayor índice de masa corporal (IMC) y los resultados peri y posoperatorios en pacientes a las que se les realizó histerectomía laparoscópica total (HLT).

Metodología: cohorte histórica de pacientes a quienes se les practicó HLT en un período de 5 años en un centro de referencia para endoscopia ginecológica. Fueron incluidas todas las pacientes con HLT y con la información completa. Las pacientes fueron divididas en tres grupos según su

* Ginecólogo Endoscopista Unidad de Endoscopia Ginecológica Clínica del Prado, Profesor de Endoscopia Ginecológica CES, Medellín (Colombia). Correo electrónico: jdcasta@hotmail.com

* Instrumentadora Unidad de Endoscopia Ginecológica Clínica del Prado, Profesora de Instrumentación Quirúrgica Universidad de Antioquia, Medellín (Colombia).

*** Ginecóloga y Obstetra CES, Magister en Epidemiología, Fellow Endoscopia Ginecológica Universidad CES, Medellín (Colombia).

****Fellow Endoscopia Ginecológica Universidad CES, Medellín (Colombia). masa corporal: IMC menor de $24,9 \mathrm{~kg} / \mathrm{m}^{2}$, normal ( $\mathrm{n}=339$ ); IMC entre $25-29,9 \mathrm{~kg} / \mathrm{m}^{2}$, sobrepeso ( $\mathrm{n}=243$ ) e IMC mayor de $30 \mathrm{~kg} / \mathrm{m}^{2}$, obesidad ( $\mathrm{n}=94)$. Se compararon el tiempo quirúrgico, las complicaciones, la pérdida sanguínea, la necesidad de transfusión, la tasa de laparoconversión y el tiempo de estancia hospitalaria. Los tres grupos se contrastaron por medio de la prueba ANOVA.

Resultados: 676 pacientes cumplieron los requisitos para el estudio. No hubo diferencias significativas en cuanto a la pérdida sanguínea, el tiempo quirúrgico, la estancia hospitalaria, la tasa de complicaciones o de laparoconversión.

Conclusión: no se encontró asociación entre el índice de masa corporal y malos resultados peri y posoperatorios.

Palabras clave: histerectomía laparoscópica total, índice de masa corporal, laparoscopia, complicaciones, tiempo quirúrgico, tiempo de hospitalización. 


\section{SUMMARY}

Objectives: evaluating the possible association between body mass index (BMI) and peri-and post-operative results in patients undergoing total laparoscopic hysterectomy (TLH).

Methodology: a historical cohort of patients was taken over a 5-year period; they had undergone TLH in a referral centre for gynaecological laparoscopy. All patients who had undergone TLH and about whom complete information was available were included. Patients were divided into three groups according to body mass as follows: BMI below $24.9 \mathrm{~kg} / \mathrm{m}^{2}$ : "normal" ( $\left.\mathrm{n}=339\right)$, BMI $25-29.9 \mathrm{~kg} / \mathrm{m}^{2}$ : "overweight" $(\mathrm{n}=243)$ and BMI greater than $30 \mathrm{~kg} / \mathrm{m}^{2}$ : “obese" ( $\left.\mathrm{n}=94\right)$. Surgical time, complications, blood loss, transfusion requirement, laparoconversion rate and length of hospital stay were compared. ANOVA was used for comparing the three groups.

Results: 676 patients met the study requirements. No significant differences regarding blood loss, operating time, hospital stay, complication rate or laparoconversion were found.

Conclusion: no association was found between BMI and poor results during peri- and postoperative periods.

Key words: total laparoscopic hysterectomy, body mass index, laparoscopy, complications, surgical time, hospital stay.

\section{INTRODUCCIÓN}

La obesidad se ha convertido en un problema de salud pública en todo el mundo. Cerca de 1.700 millones de personas en el mundo padecen sobrepeso u obesidad. Esta cifra se acerca al 25\% de la población mundial y tiende a incrementarse de manera progresiva. En Estados Unidos la obesidad se presenta en el 19,8\% de la población. ${ }^{1,2}$ Casi 40 millones de ciudadanos estadounidenses son obesos y aproximadamente la mitad de estos son mujeres. En Colombia, existen 12 millones de personas con sobrepeso u obesidad (30\%); nuestro país ocupa el tercer lugar en Latinoamérica en los índices de obesidad después de Brasil y México., ${ }^{3,4}$
La obesidad había sido catalogada como una contraindicación relativa para el abordaje laparoscópico. ${ }^{5}$ Los beneficios de la laparoscopia para diversos procedimientos han sido demostrados ampliamente. ${ }^{6}$ El desarrollo de la endoscopia ginecológica y el mejoramiento de los equipos y del instrumental han permitido que el acto quirúrgico sea llevado a cabo por esta vía en casi todas las patologías que antes eran manejadas rutinariamente con cirugía abierta. ${ }^{7}$

Hay controversia sobre si las pacientes obesas tienen mayor riesgo de resultados adversos perioperatorios. ${ }^{8} \mathrm{Si}$ bien es cierto que existen algunos problemas propios de este grupo de pacientes tales como mayor dificultad para acceder a la cavidad, riesgo de lesión de los vasos epigástricos por visualización subóptima de los mismos, dificultades para la ventilación durante la anestesia e inconvenientes para la elevación del intestino, en la actualidad el peso de la paciente o su índice de masa corporal (IMC) no son una contraindicación para la técnica.

El presente estudio pretende evaluar la posible asociación entre el mayor índice de masa corporal y los resultados peri y posoperatorios de pacientes sometidas a histerectomía laparoscópica total.

\section{MATERIALES Y MÉTODOS}

Cohorte histórica de pacientes intervenidas de HLT en la Clínica del Prado, centro de referencia en endoscopia ginecológica de la ciudad de Medellín (Colombia), entre diciembre de 2002 y abril de 2008. De 827 pacientes que se les realizó HLT se eligieron 676. Fueron incluidas todas las pacientes con HLT y con la información completa.

Las historias clínicas fueron revisadas por uno de los investigadores de acuerdo a un formulario específicamente diseñado para este estudio. Todas las cirugías fueron realizadas por patologías ginecológicas benignas por los cuatro endoscopistas de la Unidad con colaboración por parte de los residentes de Endoscopia Ginecológica de la Universidad CES que realizan su entrenamiento en la Clínica del Prado. La técnica quirúrgica fue semejante en todas las pacientes y la información extraída de las historias clínicas 
fue almacenada en una base de datos construida en Microsoft Excel ${ }^{\circledR}$ directamente por los investigadores.

El IMC se dividió en tres grupos de la siguiente manera: grupo con IMC normal: IMC menor de 24,9 $\mathrm{kg} / \mathrm{m}^{2}$; grupo con sobrepeso: IMC entre $25-29,9 \mathrm{~kg} / \mathrm{m}^{2}$ y grupo con obesidad: IMC mayor de $30 \mathrm{~kg} / \mathrm{m}^{2}$. Se compararon el tiempo quirúrgico, las complicaciones, la pérdida sanguínea, la necesidad de transfusión y el tiempo de estancia hospitalaria. El tiempo quirúrgico fue contabilizado desde el inicio del tiempo vaginal hasta la extracción de los trocares.

\section{Análisis}

Los datos fueron manejados en una base de datos de Epi Info ${ }^{\circledR} 6$ y para el análisis de la información se utilizó el programa SPSS ${ }^{\circledR} 10$. Se estableció comparación entre los tres grupos con respecto a variables como promedio de tiempo cirujano, pérdida sanguínea, porcentaje de laparoconversión, promedio de medida y peso uterino, entre otras variables; a través del análisis de varianza (ANOVA) se compararon los grupos según complicaciones a través de la prueba chi ${ }^{2}$ de independencia. Se utilizó un nivel de significación estadística de 5\%.

\section{RESULTADOS}

De las 676 historias incluidas, 339 pacientes conformaron el grupo de IMC normal (50,07\%), 243 quedaron incluidas en el grupo con sobrepeso $(35,66 \%)$ y 94 fueron catalogadas dentro del grupo con obesidad (14,26\%). El análisis de las variables propias del acto quirúrgico no evidenció diferencias significativas al comparar los tres grupos de IMC (tabla 1). No se encontraron diferencias en la presentación de complicaciones entre los grupos (tabla 2).

Las pacientes excluidas fueron comparadas con las incluidas en cuanto a las variables: tiempo quirúrgico, pérdida sanguínea, necesidad de transfusión, tiempo de estancia hospitalaria, entre otras; no se encontraron diferencias estadísticamente significativas entre ellas según los grupos de estudio, obteniendo valores de p superiores a 0,70 .

Se encontraron 5 complicaciones intraoperatorias, todas ellas en el grupo de IMC normal y ninguna en los otros grupos. No ocurrió ninguna complicación anestésica. Se presentó una lesión ureteral, la cual se identificó y se resolvió en el mismo acto quirúrgico, con anastomosis término

\begin{tabular}{|c|c|c|c|c|}
\hline Variable & $\begin{array}{c}\text { Normal } \\
(n=339)\end{array}$ & $\begin{array}{c}\text { Sobrepeso } \\
(n=243)\end{array}$ & $\begin{array}{c}\text { Obesidad } \\
(n=94)\end{array}$ & $p^{*}$ \\
\hline Promedio de tiempo cirujano (min) & 84,26 & 86,49 & 87,04 & 0,57 \\
\hline Máximo de tiempo cirujano (min) & 360 & 300 & 150 & \\
\hline Mínimo de tiempo cirujano (min) & 30 & 35 & 45 & \\
\hline Pérdida sanguínea estimada (mL) & 56,21 & 61,88 & 72,18 & 0,08 \\
\hline Necesidad de transfusión \% & 1,18 & 1,65 & 1,06 & 0,86 \\
\hline Necesidad de laparoconversión & 5 & 3 & 3 & \\
\hline Porcentaje de laparoconversión & 1,47 & 1,23 & 3,19 & 0,42 \\
\hline Promedio de tiempo internación (horas) & 19,34 & 18,84 & 18,62 & 0,73 \\
\hline Máximo de tiempo internación (horas) & 72 & 76 & 48 & \\
\hline Mínimo de tiempo internación (horas) & 6 & 6 & 6 & \\
\hline Promedio de medida útero $(\mathrm{cm})$ & 10,2 & 10,19 & 10,49 & 0,42 \\
\hline Promedio de peso útero (gramos) & 175,02 & 179,43 & 185,35 & 0,56 \\
\hline
\end{tabular}

* Prueba ANOVA 


\begin{tabular}{|l|c|c|c|c|}
\hline \multicolumn{7}{|c|}{ Tabla 2. Complicaciones quirúrgicas. } \\
\hline & $\begin{array}{c}\text { Normal } \\
(\mathbf{n}=\mathbf{3 3 9})\end{array}$ & $\begin{array}{c}\text { Sobrepeso } \\
(\mathbf{n}=\mathbf{2 4 3})\end{array}$ & $\begin{array}{c}\text { Obesidad } \\
(\mathbf{n}=\mathbf{9 4})\end{array}$ \\
\hline Complicaciones intraoperatorias & 5 & 0 & 0 & $\mathbf{0}$ \\
\hline Porcentaje de complicaciones intraoperatorias & 1,47 & 0 & 0 & NC \\
\hline Complicaciones posoperatorias & 45 & 28 & 12 & 12,77 \\
\hline Porcentaje de complicaciones posoperatorias & 13,27 & 11,52 & $0,81 *$ \\
\hline
\end{tabular}

$\mathrm{NC}$ : no calculable; $* \mathrm{Chi}^{2}$

terminal laparoscópica. Las 2 lesiones vesicales fueron detectadas y corregidas intraoperatoriamente por vía endoscópica; una lesión de vasos epigástricos y un desgarro vaginal también fueron resueltos inmediatamente.

Se presentaron 85 complicaciones posoperatorias que corresponden al 12,57\% de las cirugías. La mas frecuente fue la infección de cúpula vaginal en 32 pacientes (34,8\% del total de complicaciones posoperatorias); el hematoma de cúpula en 19 pacientes (20,6\%); la infección urinaria en 16 pacientes $(17,4 \%)$. Doce pacientes presentaron sangrado tardío de cúpula vaginal. Entre otras complicaciones se encuentran: 1 dehiscencia de sutura de cúpula, 2 infecciones en puertos, 1 obstrucción intestinal y 1 retención urinaria. No hubo ninguna muerte. 11 pacientes requirieron laparoconversión, 5 por dificultades con el colpotomizador, principalmente por no visualizarse; 4 por adherencias severas; 1 por sangrado no controlado y 1 por daño de la pinza bipolar.

\section{DISCUSIÓN}

A pesar de las observaciones iniciales que contraindicaban la histerectomía laparoscópica en pacientes con obesidad ${ }^{3}$ el presente estudio no evidenció diferencias estadísticamente significativas entre los grupos en cuanto a pérdida sanguínea, necesidad de transfusión, tiempo quirúrgico, complicaciones anestésicas, tasa de laparoconversión, ni complicaciones posoperatorias. Lo anterior es consistente con la mayoría de los trabajos publicados sobre este tópico que incluyen desde los reportes iniciales que examinaban la histerectomía vaginal asistida por laparoscopia hasta los más recientes que revisan la histerectomía radical laparoscópica. ${ }^{9-15}$ Por lo tanto hoy en día múltiples estudios respaldan el abordaje mínimamente invasivo en este grupo de mujeres. ${ }^{9-14}$

El estudio de Heinberg en 2004, ${ }^{14}$ que comparaba pacientes con IMC mayor y menor de 30, encontró diferencias estadísticamente significativas en tiempo quirúrgico y pérdida sanguínea de 18 minutos y $62 \mathrm{cc}$ respectivamente, favoreciendo al grupo con menor IMC. A pesar de las diferencias estadísticas encontradas, el significado de estas carece de importancia clínica. Por otra parte algunos autores reportan un mayor riesgo de incremento de la presión ventilatoria. ${ }^{13}$

Nuestro estudio es el primero de este tipo en nuestro país; los resultados deberán corroborarse con un estudio prospectivo especialmente diseñado para evaluar esta asociación. Existe una pérdida de poder para encontrar diferencias entre los grupos debido a que hubo un importante número de pacientes que no pudieron ser incluidas. Además, la exclusión de estos sujetos pudo incrementar el riesgo de sesgos de selección, sin embargo no parece haber tenido un impacto importante sobre la validez de los resultados presentados dado que no se encontraron diferencias estadísticas entre los pacientes incluidos y excluidos en cuanto a las variables de interés.

\section{CONCLUSIÓN}

No se encontraron diferencias entre el índice de masa corporal y los resultados peri y posoperatorios. 


\section{REFERENCIAS}

1. Mokdad AH, Bowman BA, Ford ES, Vinicor F, Marks JS, Koplan JP. The continuing epidemics of obesity and diabetes in the United States. JAMA 2001;286:1195-200.

2. Mokdad AH, Serdula MK, Dietz WH, Bowman BA, Marks JS, Koplan JP. The spread of the obesity epidemic in the United States, 1991-1998. JAMA 1999;282:1519-22.

3. World Health Organization. Global database on child growth and malnutrition. Geneva: WHO; 1997.

4. Gamboa EM, López N, Quintero DC. Prevalencia de sobrepeso y obesidad en adolescentes del municipio de Floridablanca, Colombia. MedUNAB 2007;10:5-12.

5. Gordon AG, Magos AL. The development of laparoscopic surgery. In Bailliere Clin Obstet Gynecol 1989;3:429-49.

6. Doucette RC, Scott JR. Comparison of laparoscopically assisted vaginal hysterectomy with abdominal and vaginal hysterectomy. J Reprod Med 1996;41:1-6.

7. Olive DL, Parker WH, Cooper JM, Levine RL. The AAGL classification system for laparoscopic hysterectomy. Classification committee of the American Association of Gynecologic Laparoscopists. J Am Assoc Gynecol Laparosc 2000;7:9-15.

8. Curet MJ. Special problems in laparoscopic surgery. Previous abdominal surgery, obesity, and pregnancy. Surg Clin North Am 2000;80:1093-110.
9. Frumovitz M, Sun CC, Jhingran A, Schmeler KM, Dos Reis R, Milam MR, et al. Radical Hysterectomy in obese and morbidly obese women with cervical cancer. Obstet Gynecol 2008;112:899-905.

10. Eltabbakh G, Piver MS, Hempling RE, Recio FO. Laparoscopic surgery in obese women. Obstet Gynecol 1999;94:704-8.

11. Isik-Akbay EF, Harmanli OH, Panganamamula UR, Akbay M, Gaughan J, Chatwani AJ. Hysterectomy in obese women: a comparison of abdominal and vaginal routes. Obstet Gynecol 2004;104:710-4.

12. O'Hanlan KA, Huang GS, Lopez L, Garnier AC. Selective incorporation of total laparoscopic hysterectomy for adnexal pathology and body mass index. Gynecol Oncol 2004;93:137-43.

13. O'Hanlan K, Dibble SL, Fisher DT. Total laparoscopic hysterectomy: body mass index and outcomes. Obstet Gynecol 2003;102:1384-92.

14. Heinberg EM, Crawford BL 3rd, Weitzen SH, Bonilla DJ. Total laparoscopic hysterectomy in obese versus nonobese patients. Obstet Gynecol 2004;103:674-80.

15. O’Hanlan K, Dibble SL, Fisher DT. Total laparoscopic hysterectomy for uterine pathology: impact of body mass index on outcomes. Gynecol Oncol 2006;103:938-41. 